\title{
Single flavor staggered fermions
}

\author{
Christian Hoelbling \\ Bergische Universität Wuppertal, Gaussstr. 20, D-42119 Wuppertal, Germany
}

\begin{abstract}
Based on recent work by Adams, I construct a lattice fermion operator that fully lifts the staggered flavor degeneracy. The resulting operator is of Wilson type but smaller by a factor of 4 , better conditioned and contains 3 instead of 15 doublers. It is further suggested that this operator may be used as a candidate kernel operator to an overlap construction. Prospects for practical applications and potential problems of the new discretizations are briefly discussed.
\end{abstract}

When regularizing a massless continuum Dirac operator on the lattice, one is faced with the fermion doubling problem [1, 2]. In addition to the single, physical fermion flavor, 15 doubler fermions appear at the edges of the Brillouin zone. Traditionally there have been two mutually exclusive strategies to ameliorate or solve this problem: One can either remove an exact fourfold degeneracy of the naive discretization [3, 4, 5] and reduce the doubling problem to 3 doubler species, or, alternatively, one can introduce a momentum dependent mass term to lift the degeneracy of the 15 doubler species with the physical one [6].

In this paper I give an explicit construction of a single flavor fermion operator that combines the above mentioned two approaches. It is based on recent work by Adams [7] where the two flavor case has been discussed. The construction involves adding a momentum dependent mass term as in [6], but it starts from the staggered operator [5] rather than the naive fermion operator. The resulting operator will be Wilson-like (in particular it will break chiral symmetry and require an additive mass renormalization) but smaller by a factor of 4 and with a better condition number and only 3 doubler

Email address: hch@physik.uni-wuppertal.de (Christian Hoelbling) 
fermions. It is further suggested, that this Wilson-like operator might be a suitable overlap kernel operator.

The starting point of our construction is the massless staggered fermion operator [5]

$$
D_{\mathrm{st}}=\eta_{\mu} D_{\mu}, \quad D_{\mu}=\frac{1}{2}\left(V_{\mu}-V_{\mu}^{\dagger}\right)
$$

with $\left(\eta_{\mu}\right)_{x y}=(-1)^{\sum_{\nu<\mu} x_{\nu}} \delta_{x, y}$ and $\left(V_{\mu}\right)_{x y}=U_{\mu}(x) \delta_{x+\hat{\mu}, y}$. This operator obeys a remnant chiral symmetry

$$
\left\{D_{\mathrm{st}}, \epsilon\right\}=0
$$

where $\epsilon_{x y}=(-1)^{\sum_{\mu} x_{\mu}} \delta_{x, y}$. In the spin-flavor interpretation of staggered fermions [8, 9], $\epsilon$ is identified with $\epsilon=\left(\gamma_{5} \otimes \xi_{5}\right)$.

Following [7], we first introduce a Wilson-like flavor dependent mass term

$$
M_{1}=\epsilon \eta_{5} C
$$

with $\left(\eta_{5}\right)_{x y}=\left(\eta_{1} \eta_{2} \eta_{3} \eta_{4}\right)_{x y}=(-1)^{x_{1}+x_{3}} \delta_{x, y}$ and a Laplacean-like term $C=\left(C_{1} C_{2} C_{3} C_{4}\right)_{\text {sym }}$ that is the fully symmetrized product of the $C_{\mu}=$ $\frac{1}{2}\left(V_{\mu}+V_{\mu}^{\dagger}\right)$. It is important to note that the spin-flavor structure of $M_{1}$ is

$$
M_{1} \sim\left(\mathbf{1} \otimes \xi_{5}\right)+\mathcal{O}(a)
$$

Additionally, $M_{1}$ has two crucial properties: it is hermitian and commutes with $\epsilon$. Both of these follow straightforwardly from the definitions.

Using these properties, we can immediately see that the modified staggered operator 1

$$
D_{\mathrm{A}}\left(m_{0}\right)=D_{\mathrm{st}}+r\left(1+M_{1}\right)+m_{0}
$$

with the Wilson-like parameter $r$ fulfills a $\gamma_{5}$-hermiticity like condition $D_{\mathrm{A}}\left(m_{0}\right) \epsilon=\epsilon D_{\mathrm{A}}^{\dagger}\left(m_{0}\right)$. Consequently, its non-real eigenvalues appear in complex conjugate pairs which ensures positivity of the determinant for a suitable choice of $m_{0}$. Due to its spin-flavor structure (44), the addition of $M_{1}$ in (5) will spread out the spectrum in the real direction, giving modes a mass term according to their approximate flavor chirality (cf. fig. 1(a)). It was demonstrated in [7] that this operator is a suitable overlap kernel. The resulting overlap operator obeys an index theorem with two fermion flavors [7, 10].

\footnotetext{
${ }^{1}$ Note that we have also added a mass term $r+m_{0}$ in order to shift the physical part of the spectrum to the correct position.
} 


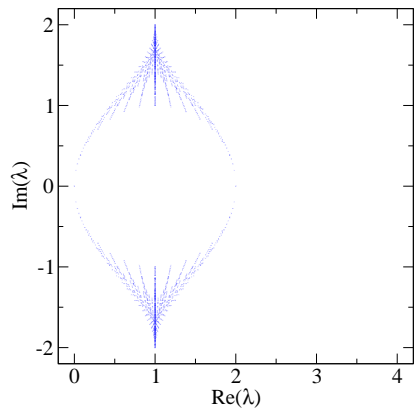

(a) Operator (5) proposed (b) in [7]

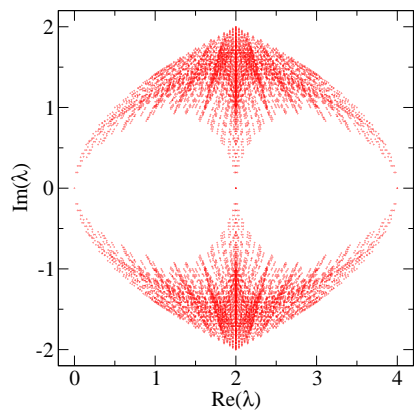

(b) Unsymmetrized operator (11)

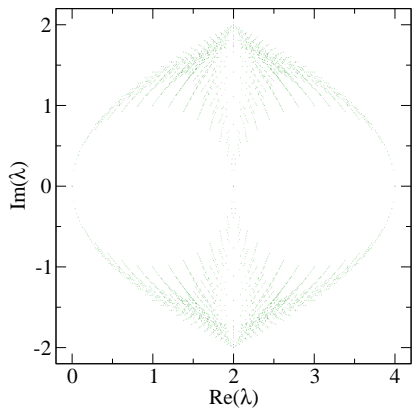

(c) Symmetrized operator (20)

Figure 1: Spectrum of various Wilson-type operators with $r=1$ and $m_{0}=0$ in the free field case on a $32^{4}$ lattice.

The fact that one is left with two fermion flavors originates in the dimension two of the positive and negative flavor chirality subspaces in four space-time dimensions. In order to lift this remaining degeneracy, an additional operator is needed, which differentiates between flavors of the same chirality. In the flavor Clifford algebra, the natural candidates are the matrices $\sigma_{\mu \nu}=i \xi_{\nu} \xi_{\mu}$. The $\sigma_{\mu \nu}$ commute with $\xi_{5}$ and can therefore simultaneously be diagonalized. Furthermore, $\sigma_{\mu \nu}$ has one eigenvalue 1 and one -1 in both the positive and negative chirality subspace. Therefore, one can choose a common diagonal basis where

$$
\xi_{5}=\operatorname{diag}(1,1,-1,-1) \quad \sigma_{\mu \nu}=\operatorname{diag}(1,-1,-1,1)
$$

In order to fully lift the flavor degeneracy, we therefore would need an additional "mass term" with the flavor structure $\left(1 \otimes \sigma_{\mu \nu}\right)+\mathcal{O}(a)$.

A candidate for such an additional term that has the correct flavor structure is given by

$$
M_{2}^{\mu \nu}=i \eta_{\mu \nu} C_{\mu \nu}
$$

with

$$
\begin{aligned}
\left(\eta_{\mu \nu}\right)_{x y} & =-\left(\eta_{\nu \mu}\right)_{x y}=(-1)^{\sum_{i=\mu+1}^{\nu} x_{i}} \delta_{x, y} \text { for } \mu<\nu \\
C_{\mu \nu} & =\frac{1}{2}\left(C_{\mu} C_{\nu}+C_{\nu} C_{\mu}\right)
\end{aligned}
$$

The spin-flavor structure of $M_{2}^{\mu \nu}$ can be made explicit by noting that

$$
\eta_{\mu \nu}=\epsilon_{\mu \nu} \eta_{\mu} \eta_{\nu} \quad\left(\epsilon_{\mu \nu}\right)_{x y}=-\left(\epsilon_{\nu \mu}\right)_{x y}=(-1)^{x_{\mu} x_{\nu}} \delta_{x, y} \text { for } \mu<\nu
$$


We have $\epsilon_{\mu \nu} \sim\left(\gamma_{\mu} \gamma_{\nu} \otimes \xi_{\nu} \xi_{\mu}\right)$ and, up to discretization terms, $\eta_{\mu} \eta_{\nu} C_{\mu \nu} \sim$ $\left(\gamma_{\mu} \gamma_{\nu} \otimes \mathbf{1}\right)$. Therefore we see that indeed $M_{2}^{\mu \nu} \sim\left(\mathbf{1} \otimes \sigma_{\mu \nu}\right)+\mathcal{O}(a)$.

It is also straightforward to check that $M_{2}^{\mu \nu}$ is hermitian and commutes with $\epsilon$. We therefore conclude that $M_{2}^{\mu \nu}$ is a valid candidate for a flavor dependent mass term and may be used for the construction of a Wilson-type operator. 2

Let us first look at the operator

$$
D_{\mathrm{k}}\left(m_{0}\right)=D_{\mathrm{st}}+r\left(2+M_{1}+M_{2}^{\mu \nu}\right)+m_{0}
$$

which is expected to fully lift the staggered flavor degeneracy. The choice of $\mu$ and $\nu \neq \mu$ is arbitrary and reflects the ambiguity of defining a single flavor in the spin-flavor representation. Note that $\left[M_{2}^{\mu \nu}, \epsilon\right]=0$ together with the hermiticity of $M_{2}^{\mu \nu}$ implies $D_{\mathrm{k}}(m) \epsilon=\epsilon D_{\mathrm{k}}^{\dagger}(m)$. Consequently, non-real eigenvalues of $D_{\mathrm{k}}(m)$ also appear in complex conjugate pairs.

In fig. 1(b), the spectrum of $D_{\mathrm{k}}(0)$ is plotted for the free case. Note that the multiplicity of the real eigenmodes is $(4,8,4)$ as compared to $(8,8)$ for the free $D_{\mathrm{A}}(0)$ displayed in fig. 1(a), indicating that the flavor degeneracy has indeed been lifted.

In order to investigate the symmetries of the single flavor operator (11), we proceed to investigate the transformation properties of $M_{2}^{\mu \nu}$ under the the staggered symmetries [11]. Note, that in addition to breaking the flavor chiral symmetry $U(1)_{\epsilon}, M_{2}^{\mu \nu}$ also breaks the discrete shift, axis reversal and hypercubic rotational symmetries. Explicitly, we have

$$
M_{2}^{\mu \nu} \rightarrow M_{2}^{\mu \nu} \cdot\left\{\begin{array}{rc}
-1 & \text { for } \\
1 & \text { else }
\end{array}\right.
$$

under both shift and axis reversal symmetries along the direction $\rho$ and

$$
M_{2}^{\mu \nu} \rightarrow R_{\mu \alpha}^{(\rho \sigma)} R_{\nu \beta}^{(\rho \sigma)} M_{2}^{\alpha \beta}
$$

\footnotetext{
${ }^{2}$ Note, that in two dimensions $M_{2}^{12}$ is the unique term required for fully lifting the twofold staggered flavor degeneracy (up to a trivial sign). Furthermore, one can obtain the flavor chiral mass term $M_{1}$ as the antisymmetrized product of the $M_{2}^{\mu \nu}$ via

$$
M_{1}=-\frac{1}{4 !} \epsilon_{\alpha \beta \mu \nu} M_{2}^{\alpha \beta} M_{2}^{\mu \nu}
$$


under a rotation of the $\rho$ into the $\sigma$ direction, where the rotation matrix $R^{(\rho \sigma)}$ is explicitly given by

$$
R_{\mu \alpha}^{(\rho \sigma)} x_{\alpha}=\left\{\begin{array}{rll}
x_{\sigma} & \text { for } & \mu=\rho \\
-x_{\rho} & \text { for } & \mu=\sigma \\
x_{\mu} & \text { else } &
\end{array}\right.
$$

Note that rotational transformations (13) can generate an $M_{2}^{\alpha \beta}$ term with arbitrary $\alpha$ and $\beta \neq \alpha$ out of any given $M_{2}^{\mu \nu}$. This suggests that instead of adding $M_{1}$ and a single $M_{2}^{\mu \nu}$ to the staggered operator as in (11), one might instead take a more symmetric linear combination of the different $M_{2}^{\mu \nu}$.

We proceed to investigate the following linear combinations

$$
\begin{gathered}
M_{s}=\frac{1}{\sqrt{3}}\left(s_{12}\left(s_{1} s_{2} M_{2}^{12}+s_{3} s_{4} M_{2}^{34}\right)\right. \\
+s_{13}\left(s_{1} s_{3} M_{2}^{13}+s_{4} s_{2} M_{2}^{42}\right) \\
\left.+s_{14}\left(s_{1} s_{4} M_{2}^{14}+s_{2} s_{3} M_{2}^{23}\right)\right)
\end{gathered}
$$

where the $s_{\mu}= \pm 1$ and $s_{\mu \nu}= \pm 1$ are arbitrary sign prefactors. One can check that the effect of both shift translation and axis inversion in $\rho$ direction is a single sign flip $s_{\rho} \rightarrow-s_{\rho}$. Similarly, the effect of a single hypercubic rotation is to flip the sign of one single $s_{\mu \nu}$ (cf. table 1). As a result, $M_{s}$ is invariant under the following discrete symmetries:

1. Diagonal shift:

$$
x \rightarrow x+\hat{1} \pm \hat{2} \pm \hat{3} \pm \hat{4}
$$

2. Shifted axis reversal:

$$
x_{\mu} \rightarrow-x_{\mu}+\hat{\mu}
$$

3. Double rotation:

$$
x \rightarrow R^{(\mu \nu)} R^{(\rho \sigma)} x
$$

with $(\mu, \nu, \rho, \sigma)$ any permutation of $(1,2,3,4)$

These symmetries form a subrgoup of the original discrete staggered symmetries shift, axis reversal and hypercubic rotation [11].

Up to discretization effects, the flavor structure $\xi^{(s)}$ of $M_{s}$ is given by a linear combination of the $\sigma_{\mu \nu}$, such that in the common diagonal basis one can write

$$
\xi_{5}=\operatorname{diag}(1,1,-1,-1) \quad \xi^{(s)}=\left\{\begin{array}{l}
\operatorname{diag}(2,-2,0,0) \\
\operatorname{diag}(0,0,2,-2)
\end{array} \quad\right. \text { or }
$$




\begin{tabular}{cccc|c}
\multicolumn{4}{c|}{$(\rho, \sigma)$} & sign flip \\
\hline$(1,4)$ & $(2,3)$ & $(3,1)$ & $(2,4)$ & $s_{12} \rightarrow-s_{12}$ \\
$(1,2)$ & $(3,4)$ & $(4,1)$ & $(3,2)$ & $s_{13} \rightarrow-s_{13}$ \\
$(1,3)$ & $(4,2)$ & $(2,1)$ & $(4,3)$ & $s_{14} \rightarrow-s_{14}$
\end{tabular}

Table 1: Explicit table of sign flips in (15) under the various hypercubic rotations $R^{(\rho \sigma)}$

which indicates, that a single $M_{s}$ alone should fully lift the flavor degeneracy. 3 I therefore propose the following symmetrized Wilson-like operator

$$
D_{\mathrm{s}}\left(m_{0}\right)=D_{\mathrm{st}}+r\left(2+M_{s}\right)+m_{0}
$$

Again, one can demonstrate that $D_{\mathrm{s}}(m) \epsilon=\epsilon D_{\mathrm{s}}^{\dagger}(m)$ so that the eigenvalues of $D_{\mathrm{s}}(m)$ also appear in complex conjugate pairs. The spectrum of the free $D_{\mathrm{s}}(0)$ is plotted in fig. $1(\mathrm{c})$. One can see that it is similar to the one of (11) (cf. fig. 1(b) with the same multiplicity of real eigenmodes $(4,8,4)$. Note however that the spectrum does display a higher degeneracy of the eigenmodes which reflects the improved symmetry properties of the free (20) as compared to the free (11).

In the interacting theory (20) will receive radiative corrections. The structure of these corrections will be restricted by the symmetries (16, 18) (in addition to the usual gauge and baryon number symmetries). These symmetries form a subgroup of the discrete staggered symmetries [11]. The counterterm structure of the single flavor operator (20) will be the one of the 2-flavor operator (5) plus additional local terms that break the hypercubic rotational symmetry but do preserve the double rotation symmetry (18).

The dimension 3 counterterms that can appear are therefore the scalar $\bar{\chi} \chi$, the 2-flavor mass term $\bar{\chi} M_{1} \chi$ and all of the $\bar{\chi} M_{\mathrm{s}} \chi$. As in the case of Wilson fermions, $\bar{\chi} \chi$ necessitates an additive mass renormalization. To leading order, the effect of the 2-flavor mass term $\bar{\chi} M_{1} \chi$ will be a relative shift of the physical and doubler branches of the spectrum as one can see from (19). Mixing among the different $\bar{\chi} M_{\mathrm{s}} \chi$ on the other hand will lead to a renormalization of the flavor structure. This is evident from the fact that the different $\xi^{(s)}$ do not all commute among each other. The flavor assignment to leading order will no more be given by the $\xi^{(s)}$ but instead by a linear combination of

\footnotetext{
${ }^{3}$ Note that here the two flavors of one chirality receive an opposite mass term while both flavors of the other chirality remain unaffected up to discretization effects.
} 
them 4 The radiative corrections will therefore modify the particular linear combination of the four degenerate flavors of the underlying staggered operator that will end up in the physical branch of the spectrum. But since the details of the flavor assignment are arbitrary in any case and do not carry any further physical significance, the renormalized flavor assignment is as good as the bare one and there is no need to undo this mixing. Apart from this renormalized flavor assignment, the radiative corrections will obviously move the relative positions of the physical and doubler branches. This however can be absorbed by a further additive mass renormalization as long as a clear separation between the physical and doubler branches is maintained (which is expected to be the case outside the strong coupling regime).

Note that radiative corrections are generally expected to be suppressed by UV-filtering (and Symanzik improving) the fermion operator in much the same way as it is the case for staggered [12, 13] and Wilson fermions [14, 15].

A further detailed investigation of this issue is beyond the scope of this paper but will be an essential ingredient in judging the practical usefulness of (20).

We now proceed to use $D_{\mathbf{s}}(m)$ as the kernel of an overlap operator [16]. We define a massless overlap operator

$$
D_{1}=\rho\left(1+\epsilon \operatorname{sign}\left(\epsilon D_{\mathrm{s}}(-\rho)\right)\right)
$$

that is conjectured to describe a single flavor if $\rho$ is chosen properly, i.e. in a range that extends to $\rho \in(0,2)$ in the free case. Whether (21) constitutes a valid overlap operator remains to be seen. Specifically, the counterterm structure and the question of locality and a spectral gap [17] in the hermitian kernel operator $\epsilon D_{\mathrm{s}}(-\rho)$ require further investigations.

Beyond the remaining conceptual questions it is interesting to speculate about the usefulness of either (20) or (21) for lattice QCD calculations. The obvious advantage of (20) as compared to a standard Wilson operator are its smaller size (by a factor of 4) and the reduced condition number of $D^{\dagger} D$ (another factor of 4 for $m_{0}=0$ in the free case). This leads to a reduction in storage requirement by a factor of 4 and to a naively estimated speedup factor of $\sim 16$ for conjugate gradient inversions or the construction of the overlap operator (21). Note that in contrast to the staggered fermion case these improvements originate in the huge reduction of UV modes (by eliminating 12

\footnotetext{
${ }^{4}$ Note however, that all linear combinations of the $\xi^{(s)}$ do still commute with $\xi_{5}$.
} 
of the 15 doubler fermion species) and do not come at the price of quadrupling the IR modes in the physically relevant branch of the spectrum.

There are also two obvious disadvantages of (20) as compared to a standard Wilson operator. The first one is the appearance of two-hop terms in the Wilson-like operator (20). These terms could lead to a slower numerical implementation when compared to strictly one-hop operators. It is however interesting to note, that the additional gauge links needed for the two-hop terms are exactly the same gauge links one needs for the construction of a clover-term [18] that can be used to $O(a)$ improve a Wilson operator. One therefore expects on the one hand that the speed loss due to 2-hop terms would be approximately equal to the speed loss by including a clover term (which is typically about 30\%) and on the other hand that the inclusion of a clover term into (20) would essentially be free.

A second concern is that (20) was constructed using the staggered spinflavor basis and will therefore inherit the nonlocal definition of the spin matrices. In contrast to staggered fermions however, there will be no significant flavor mixing due to the Wilson-like lifting of the flavor degeneracy. Doubler states will quickly die out in correlation functions and will not have to be disentangled as for staggered fermions. I therefore do not expected that e.g. ground state hadron masses or simple matrix elements like decay constants or $B_{K}$ will be harder to obtain than with Wilson fermions. It might however turn out to be difficult to extract some short distance observables with nontrivial spin structure in this formulation.

\section{Acknowledgments}

I would like to thank Maarten Golterman and David Adams for valuable comments on the manuscript and Stephan Dürr for helpful discussions. In reaction to a previous version of this manuscript I was informed that Philippe de Forcrand, Aleksi Kurkela and Marco Panero have presented a spectrum

plot of a single flavor staggered operator at a recent workshop [19]. Although no further details were given, the plot closely resembles fig. 1(b). This work was supported by the DFG grant SFB-TR 55 .

\section{References}

[1] L. H. Karsten and J. Smit, Nucl. Phys. B 183, 103 (1981). 
[2] H. B. Nielsen and M. Ninomiya, Nucl. Phys. B 185, 20 (1981) [Erratumibid. B 195, 541 (1982)].

[3] J. B. Kogut and L. Susskind, Phys. Rev. D 11, 395 (1975).

[4] T. Banks, L. Susskind and J. B. Kogut, Phys. Rev. D 13, 1043 (1976).

[5] L. Susskind, Phys. Rev. D 16, 3031 (1977).

[6] K. G. Wilson, in New Phenomena in Subnuclear Physics (Erice, 1975), ed. A. Zichichi (New York, Plenum, 1975).

[7] D. H. Adams, arXiv:1008.2833 [hep-lat].

[8] F. Gliozzi, Nucl. Phys. B 204, 419 (1982).

[9] H. Kluberg-Stern, A. Morel, O. Napoly and B. Petersson, Nucl. Phys. B 220, 447 (1983).

[10] D. H. Adams, Phys. Rev. Lett. 104, 141602 (2010)

[11] M. F. L. Golterman and J. Smit, Nucl. Phys. B 245, 61 (1984).

[12] T. Blum et al., Phys. Rev. D 55, 1133 (1997) arXiv:hep-lat/9609036.

[13] K. Orginos, D. Toussaint and R. L. Sugar [MILC Collaboration], Phys. Rev. D 60, 054503 (1999) arXiv:hep-lat/9903032.

[14] T. A. DeGrand, A. Hasenfratz and T. G. Kovacs, Nucl. Phys. B 547, 259 (1999) arXiv:hep-lat/9810061.

[15] S. Capitani, S. Durr and C. Hoelbling, JHEP 0611, 028 (2006) arXiv:hep-lat/0607006.

[16] H. Neuberger, Phys. Lett. B 417, 141 (1998)

[17] M. Golterman and Y. Shamir, Phys. Rev. D 68, 074501 (2003)

[18] B. Sheikholeslami and R. Wohlert, Nucl. Phys. B 259, 572 (1985).

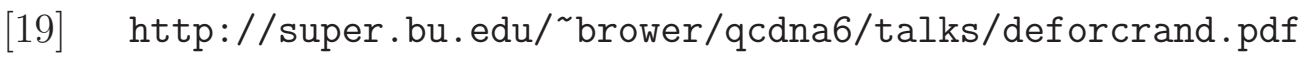

